\title{
Les assemblages mécaniques - Évolutions et perspectives
}

\section{Mechanical Assemblies - Trends and Perspectives}

Reçu le 2 octobre 2018

Les exigences de performances, en termes de fiabilité, sécurité, économie d'énergie, et respect de l'environnement, imposées à la construction mécanique d'aujourd'hui, passent par l'optimisation de l'ensemble du cycle d'un produit, depuis sa conception jusqu'à sa tenue en service.

En particulier, afin d'atteindre les objectifs de performance, de réduction des coûts de production et de diminution des dépenses de fonctionnement, les concepteurs ont recours à l'utilisation de matériaux et de composants de natures différentes qu'il s'agit d'assembler.

Cette démarche conduit à rechercher, optimiser voire imaginer des procédés d'assemblages mécaniques répondant aux conditions de service de plus en plus exigeantes.

La maîtrise et la bonne connaissance de ces procédés constituent des points essentiels pour leur intégration efficace et optimale dans les structures mécaniques industrielles.

C'est pourquoi, le développement d'outils numériques capables de modéliser ces assemblages constituent souvent un point clé pour l'aide à la conception et à l'innovation. Ces outils viennent compléter ceux existants ainsi que l'expérience métier des concepteurs.

Pour progresser vers ces buts, de nombreux travaux sont en cours dans les thématiques suivantes:

- l'évolution des procédés et des démarches d'assemblage;

- l'application des méthodes de conception avancées;

- la dynamique du contact dans les liaisons mécaniques;

- la prévision du comportement en service;

- les techniques de caractérisation des assemblages;

- le développement de solutions optimisées, nouvelles ou innovantes.

Les colloques sur «Les assemblages mécaniques-évolutions et perspectives », organisés par Supméca Alumni, se sont fixé comme objectif de faire le point sur ces thématiques de façon bisannuelle.

Le présent numéro de la revue Matériaux $\&$ techniques illustre la démarche, en rassemblant des articles issus de conférences données lors de l'édition 2017 du Colloque [1-8] et évalués par des experts que nous tenons à remercier.

\section{Références}

1. L. Gornet, R. Hamonou, F. Jacquemin, S. Auger, P. Chalandon, Détermination de la souplesse d'un assemblage de composites boulonnés à l'aide d'une démarche d'homogénéisation, Matériaux \& Techniques 106, 302 (2018)

2. J. Dhers, Assemblages non soudés dans les réacteurs nucléaires, Matériaux \& Techniques 106, 303 (2018)

3. J.-F. Rit, S. Meunier, J. Ferrari, D. Hersant, J.-P. Mathieu, Prédire correctement l'effet des chocs thermiques sur l'assemblage corps couvercle d'un robinet, Matériaux \& Techniques 106, 304 (2018)

4. F. Goldspiegel, P. Michel, J.-B. BIumenfeld, J.-B. Szenda, K. Mocellin, Influence des effets dynamiques et thermiques sur l'assemblage par clouage-Étude numérique et expérimentale, Matériaux \& Techniques 106, 305 (2018)

5. C. Delcher, Nouvelle approche pour la caractérisation du dévissage et desserrage des assemblages vissés, Matériaux \& Techniques 106, 306 (2018)

6. F. Pichoff, M. Kummel, M. Schiff, Dynamic vibration testing of fasteners: fastener self-loosening theory, vibration testing practical applications, comparison of the international standards and recommendations on how to set-up a meaningful testing protocol, Matériaux \& Techniques 106, 307 (2018)

7. J. Redford, H.-P. Lieurade, M. Gueguin, F. Hafid, C. Yang, J.M. Ghidaglia, Modélisation numérique du phénomène de fretting-fatigue intervenant dans le vieillissement des conducteurs de lignes aériennes, Matériaux \& Techniques 106, 308 (2018)

8. F. Pennec, B. Tikri, S. Bergamo, M. Duchet, B. Weber, J.-L. Robert, Experimental and numerical investigation of the overload effect on fatigue behaviour of spot-welded steel sheets, Matériaux \& Techniques 106, 309 (2018)

Pierre Chalandon

Alain Daidie

Henri-Paul Lieurade ${ }^{*}$

* e-mail: lieuradehp@gmail.com

Citation de l'article : Pierre Chalandon, Alain Daidie, Henri-Paul Lieurade, Numéro thématique sur les assemblages mécaniques, Matériaux \& Techniques 106, 301 (2018) 